\title{
A RUNNING-IN MODEL FOR THE RECIPROCATING SLIDING OF NYLON 6.6 AGAINST STAINLESS STEEL
}

\author{
Z. ZALISZ \\ Mechanical Engineering Instilule, Polylechnic of Opole, ul. Z.S.P.5, 45.233 Opole \\ (Poland) \\ P. H. VROEGOP and R. BOSMA
}

Department of Mechanical Engineering, University of Twente, P.O. Box 217, 7500 AE Enschede (The Netherlands)

(Received April 9, 1987; accepted August 4, 1987)

\section{Summary}

Results are presented of investigations of the running-in period in reciprocating sliding of a semicrystalline polymer, Nylon 6.6, on metal. A correlation was established between the changes in transfer film geometry and both the friction coefficient and the wear rate variations during a single stroke, as well as with sliding time. The role of wear products in running-in was found to be the determining factor. In order to avoid any unspecified influence of frictional heat variation on the sliding process, the counter specimen temperature was kept constant. Significant correlations, between both local and mean coefficients of friction and local and total areas of transfer film respectively, were found. The latter relation shows the existence of two different types of running-in process which depend on whether, initially, adhesive or abrasive wear mechanisms prevail. In both cases, the building up of the transfer film together with roll-like wear particles create several types of interactions between the rubbing bodies. These interactions, subsequently called subprocesses, have their own different specific coefficients of friction and can exist on the sliding track solely or in combination. They also change their distribution over the track and finally create a variation in the mean friction coefficient with time. This variation is expressed as a function of the total area fraction of transfer film spreading with time. It is also shown that changes in wear rate coincide with variations in mean friction coefficient. The presented model can also be extended qualitatively to other semicrystalline polymers.

\section{Introduction}

Sliding friction and wear of polymers on metals, under certain conditions, lead to material transfer from the polymer to the metallic counterface. Most authors agree that the transferred material takes part in the 
sliding operation and produces significant changes in the friction coefficient and in the wear rate. Frictional transfer, as the most important characteristic of adhesive wear of polymers, is the subject of extensive and multidisciplinary studies. Most of these studies are concerned with multiple pass-steady state conditions. The first period of sliding, called "unsteady state wear" or "running-in" is often only briefly mentioned or even ignored as a temporary irrevocable stage. However, some more recent publications concern transfer film formation, identified with the running-in phase of sliding. These investigations have been performed on several types of polymers sliding on metals (usually) in unidirectional motion. From these papers the following view on transfer film formation emerges.

There are three effects that contribute to material transfer: the deformation of surface asperities under load; the fracture of material in the substrate; the adherence of this material to the other surface [1]. They depend on the type of wear mechanism; abrasion for rough or adhesion for smooth counterfaces. If abrasion is the dominant type of wear process, the wear particles are produced by tearing of the material, (locally) softened by frictional heating from the polymer surface. If a particle strongly adheres to the counterface, a transfer film begins to form.

Subsequent transfer occurs preferentially on to the first adhered particles, so bands build up by the agglomeration of wear particles [2]. The bands grow in length and widen as sliding progresses. Finally, when the transfer film fully covers the sliding track, the transition from unsteady to steady state wear occurs.

In the case of an adhesive wear process, the building up of the transfer film seems to be similar to that described above but wear arises from the shear and fracture of adhesively bonded junctions. The general features of the fully developed transfer film depend on the severity of sliding which is defined in terms of the product $\mu L V$, where $\mu$ is the coefficient of friction, $L$ is the applied load and $V$ is the sliding speed [1 - 4]. At low $P V$ values the film is not continuous and consists of a relatively thick and hard agglomeration of adhered wear particles. Al high $P V$ values the continuous and very thin film heats up and behaves more as a viscous liquid. During running-in the thickness of the transfer film increases with sliding time until a maximum and constant value is reached. The time to reach it depends strongly on the initial roughness of the metallic counterface. Transfer is easier with smoother counterfaces where more intimate contact facilitates adhesion $[4,5]$. The final limiting thickness of the transfer film is probably a characteristic feature of each rubbing pair and the operating variables $[2,3,6]$. It is determined by the balance between the removing action of the front edge of the polymer body and the replenishment of the polymeric film by material transfer $[4,6]$. Obviously, drastic changes in counterface surface topography $[3,5]$ and in the material constants of the transfer film lead to changes in friction coefficient and wear rate.

It is usually recognized that the initial wear rate is very high and depends significantly on the initial roughness of the counterface. Gradual 
build up of the transfer film during running-in produces a decrease in the wear rate which levels off at a certain constant value when the steady state sliding regime is entered. The decrease in wear rate is explained by the lower surface roughness of the transfer film covering the counterface or by a Cransilion from abrasive wear to adhesive wear or both $[4,7,8]$.

It has been found that when a transfer film is formed the wear rate is lower for a high severity of sliding than for low severity [2] but it is not clear whether the speed or the load is more responsible. Some authors have concluded that sliding speed has no apparent effect on wear rate [8] whereas others have argued that the wear rate is independent of the applied load $[4,7]$ or, on the contrary, that the wear rate increases with increasing load $[5,6,9]$.

Generally, it is concluded that, as far as the coefficient of friction is concerned, it increases with sliding distance (more rapidly for smooth counterfaces) and remains approximately constant when the thickness of the transfer film becomes constant $[2,6,8,9]$. This limiting friction coefficient $\mu_{1}$ is lower for low severity conditions, corresponding to the thick, hard and discontinuous transfer film, and higher for a high severity of sliding when the transfer film behaves like a homogeneous viscous liquid [2].

The influence of speed on the final limiting friction coefficient $\mu_{1}$, observed in the steady state regime, is not clear. It was found that $\mu_{1}$ increases with increasing speed $[2,8]$, especially for a low severity of sliding, but it is independent of the speed for severe sliding. It was also found that $\mu_{1}$ increases with decreasing speed [2] and that it decreases with increasing load for most types of polymers [9]. The initial roughness of the counterface does not seem to affect the friction coefficient in the steady state regime but plays a very important role in sliding, influencing the running-in period $[4,5,9]$. Only one publication discusses running-in during the reciprocating sliding of polymers on metal [7]. It emerges from this paper that, in principle, running-in in reciprocating sliding is generally similar to that in unidirectional motion. However, some differences do exist. Wear debris is different in shape, compared with that observed in the unidirectional tests. Frequently, it takes the form of "rolls" and plays a more important part in the wear process. The transfer film is uneven in thickness, increasing at the ends of the track compared with that at the centre. The wear rate decreases with time and finally reaches a value much lower than that found in unidirectional motion. The friction coefficient rises with the formation of the transfer film until it reaches a more or less constant value.

This description of the running-in period in polymer sliding indicates that a particularly significant factor in the sliding process is the temperature of the rubbing contact, resulting from a balance between frictional heat production and its dissipation. Some aspects of the contact temperature in sliding have been considered by several authors $[2,3,5,8,10]$ but the temperature itself was not an operational variable; rather the result of the sliding process. 
The descriptive model for running-in presented above is insufficient to explain the variations in the local friction coefficient with the polymeric slider position during a single stroke of reciprocating motion. It also does not explain the variations in the relation between local friction coefficient and specimen position with sliding time or number of strokes. These were fixed on photographs during introductory tests for reciprocating sliding of Nylon 6.6 against stainless steel on the $S$ tribometer [11].

The general goal of the work presented in this paper is an explanation of the reasons for the variations in friction in reciprocating sliding of a polymer on a metal before the steady state regime is reached.

\section{Experimental procedure}

\subsection{The specimen}

The Nylon 6.6 used in the experiments was Akulon S-240C manufactured in the form of a large plate $3.3 \mathrm{~mm}$ thick. All wear strips were machined from one plate with a working surface of $11 \mathrm{~mm} \times 3.3 \mathrm{~mm}$. The bearing surface of the strip was cut on a microtome to obtain a high initial smoothness and flatness. Before use the specimens were cleaned ultrasonically in carbon tetrachloride.

\subsection{The counterspecimen}

Stainless steel counterfaces were machined from austenitic stainless steel RVS 316 . They were plates of $58 \mathrm{~mm} \times 18 \mathrm{~mm} \times 5 \mathrm{~mm}$. All wear surfaces were prepared by surface grinding and/or lapping to the required roughness. A hole was drilled into the side of the plates to locate a thermocouple in the centre. Before use, the counterfaces were cleaned ultrasonically in carbon tetrachloride.

\subsection{The apparatus}

Friction and wear tests were carried out on the $\mathrm{S}$ tribometer shown in Fig. 1. The apparatus executes a sinusoidal reciprocating motion of a specimen on the stationary counterface of amplitude $46 \mathrm{~mm}$. The specimen (1) is fixed in a holder (2), held in a loading arm (3), connected to the slider (4). The pivot of this loading arm lies in the plane of the sliding contact. The slider is driven by a crank mechanism (5) connected by a belt transmission to a motor with a continuously variable speed. The specimen is loaded through a short spindle (7) by a pressurized air bellows (6) fixed on top of the slider. The slider moves in a plane parallel to the counterface through a system of four roll blocks (8) guiding two cylindrical bars (9) fixed to the slider. An inductive displacement transducer (10) is placed in a holder (11) fixed to the front of the slider. The measuring spindle of the transducer touches the cantilever (12), being part of the loading arm. The counterface (13) is held in an aluminium holder (14) which is fixed to the heat exchanger (15). The heat exchanger is connected to a 


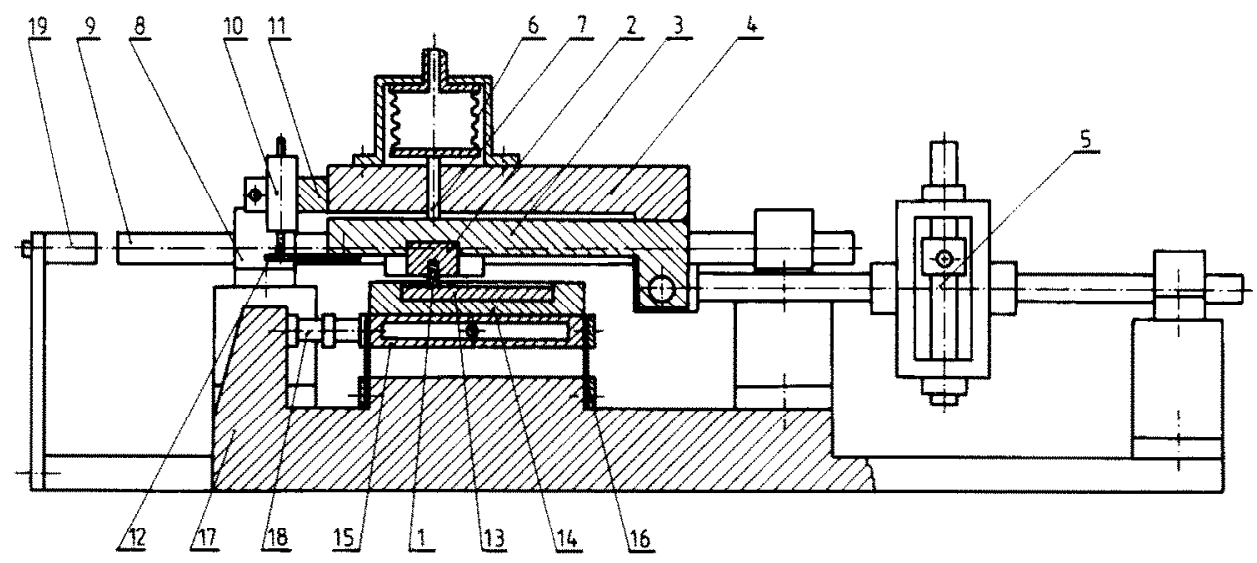

Fig. 1. Schematic arrangement of the $\mathrm{S}$ tribometer.

thermostatically controlled oil bath and is supported by two steel blade springs (16) fixed to the frame (17) of the tribometer. These springs only allow movement parallel to the direction of sliding. This movement is restricted by a piezoelectric force transducer (18) fixed between the heat exchanger and the frame of the tribometer. An electromagnetic transducer (19) is used for triggering and counting the number of cycles.

A diagram of the electrical measuring system is presented in Fig. 2. It consists of four measuring blocks.

Block I. Temperature measurement by means of $\mathrm{Cu}-\mathrm{CuNi}$ thermocouples of (1) the counterface temperature $T_{\mathrm{c}}$ in its central point $2.5 \mathrm{~mm}$ below the surface, (2) the holder temperature $T_{\mathrm{h}}$ in its centre $1 \mathrm{~mm}$ below the bottom surface, (3) the oil temperature $T_{\mathrm{o}}$ of the thermostatically controlled bath.

The thermocouples are directly connected to the recorder.

Block II. Frictional force $F_{\mathrm{f}}$ measurement, where the output from the force transducer is fed into the charge amplifier. The output signal from the amplifier is simultaneously used for (1) visualization on the oscilloscope of the coefficient of friction $\mu_{\mathrm{x}}$ as a function of the relative position $x$ of the slider, (2) recording of the maximum value of the friction coefficient $\hat{\mu}_{\mathrm{x}}$, measured as the peak-to-peak value of the friction signal during one cycle, (3) recording of the first harmonic of the signal measured using an adjustable band pass filter. Appendix A shows that this value is proportional to the frictional work per cycle and thus to the mean coefficient of friction $\bar{\mu}$, (4) spectral analysis of the variations in the frictional force for frequencies up to $100 \mathrm{kHz}$, on a spectrum analyser.

Block III. Measurement of the wear $\Delta h$ of the specimen by a displacement transducer connected to the recorder through an amplifier and a low pass filter.

Block IV. Measurement of the frequency of the reciprocating motion and the number of cycles in sliding time, where the pulse output from 


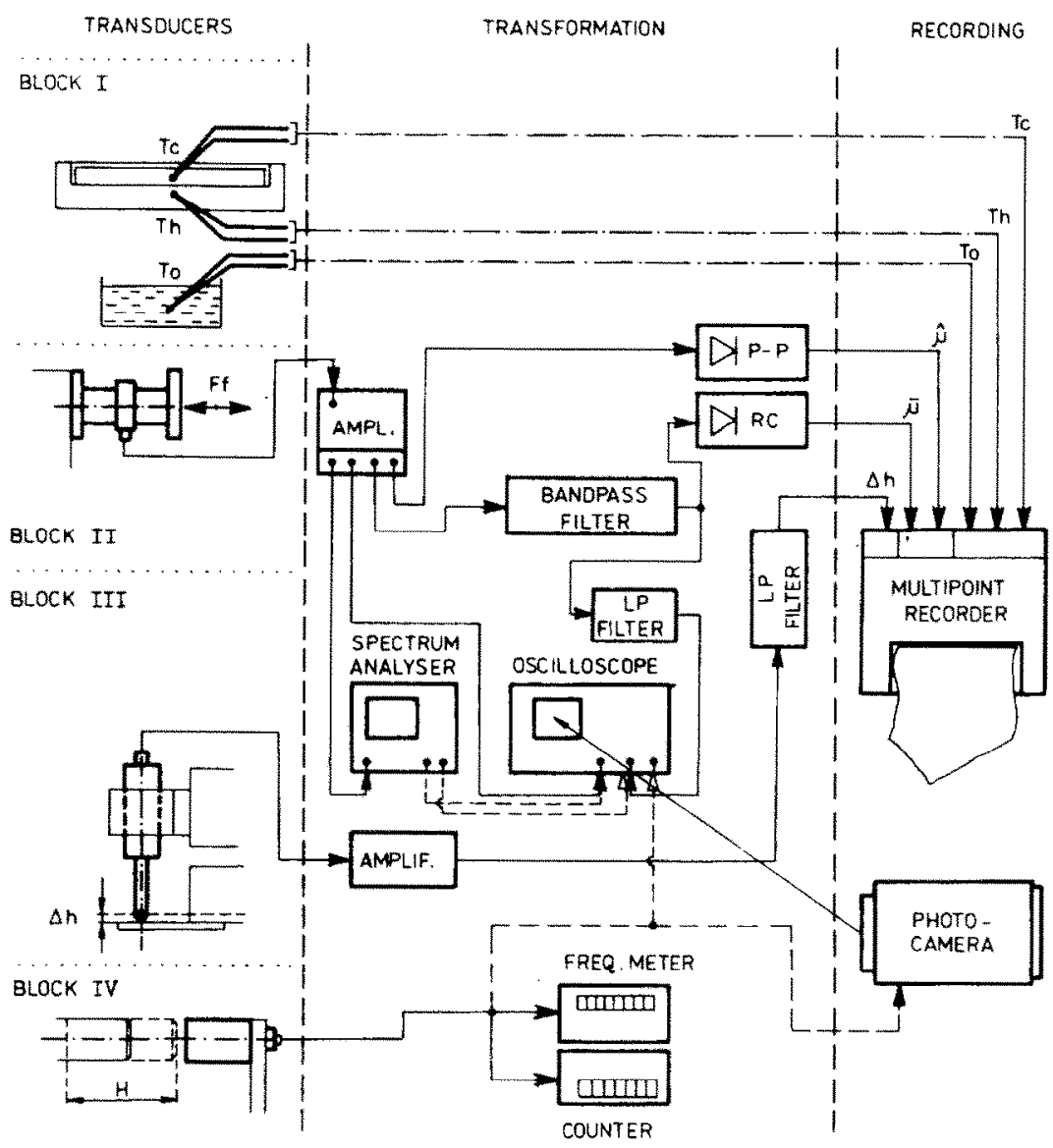

Fig. 2. Measuring system of the $\mathrm{S}$ tribometer.

the electromagnetic pick-up is converted in the frequency meter and in the electromechanical counter.

The three temperatures, the maximum and mean value of the coefficient of friction, and the displacement (wear) of the polymeric strip are recorded as a function of sliding time (distance) by means of a 12-channel multipoint recorder.

The operational variables are as follows: the normal load $L$; the average sliding speed $\bar{V}$; the temperature of the plate $T_{c}$; the initial roughness of the plate working surface $R_{\mathrm{a}}$. Additionally, optical microscopy was employed for examination of the specimens' working surfaces and wear particles. A profilometer and an image processing system were both used for measurement of the transfer film geometrical features.

\section{General assumptions}

It has been found in introductory tests that, under some conditions, the frictional force varies strongly over the length of sliding track, giving 
(on an oscilloscope) a characteristic shape of the curve $\mu_{\mathrm{x}}=\mathrm{f}(x)$. This curve-shape, as well as the mean coefficient of friction $\bar{\mu}$, varies with time. Three examples are shown in Fig. 3. The figure clearly shows a lack of any direct correlation between the local friction coefficient and the actual sliding speed. These variations, however, seem to be related to variations in the transfer film over the sliding track.

It is assumed that the local coefficient of friction is either related to the local thickness of the transfer film or to the local coverage of the plate with transferred polymer, or to both. Consequently, the mean coefficient of friction should be related either to the average thickness of the transfer film or to the total area of film covering the sliding track or to both. Investigations were divided into two parts. Firstly, the relation between the local coefficient of friction and the local geometry of the transfer film was investigated, and secondly, the relation between the mean friction coefficient and the total area of a transfer film. These results, together with observations of the plate surface and the wear debris, were used to determine a model for the running-in period of reciprocating sliding.
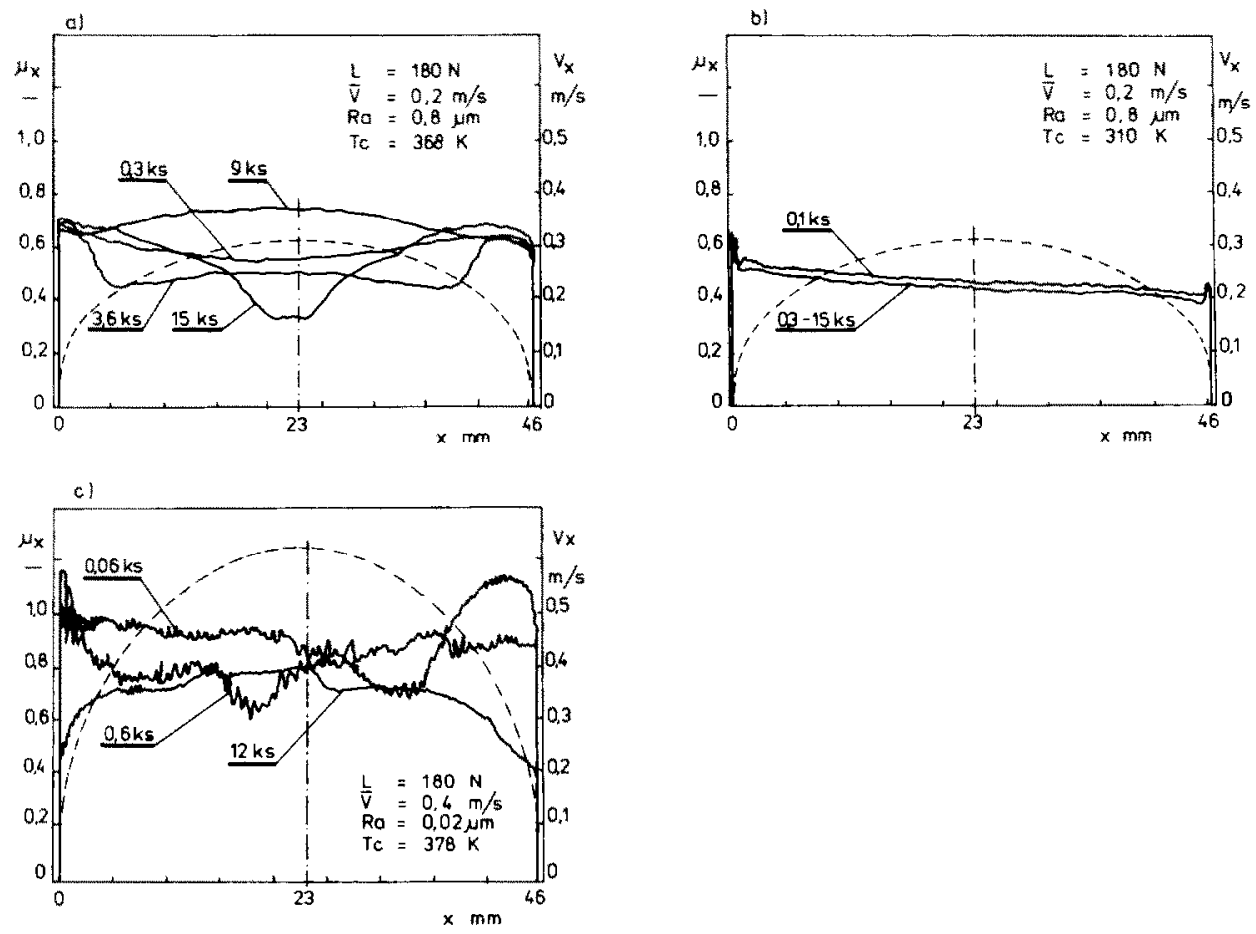

Fig. 3. Examples of the variation with time of the local coefficient of friction $\mu_{x}$ as a function of the position $x$ of the strip. Curves are photographed from the oscilloscope: $-\ldots, \mu_{\mathrm{x}}=\mathrm{f}(x) ;-\cdots$, local sliding speed $V_{\mathrm{x}}$ distribution. 


\section{Results and discussion}

\subsection{Local friction coefficient}

The experimental conditions of the four series of experiments are summarized in Table 1 . Each set of experiments was repcated three times.

TABLE 1

Experimental conditions

\begin{tabular}{lllll}
\hline Parameters & $\begin{array}{l}T_{\mathbf{c}} \\
(\mathrm{K})\end{array}$ & $\begin{array}{l}R_{\mathrm{a}} \\
(\mu \mathrm{m})\end{array}$ & $\begin{array}{l}L \\
(\mathrm{~N})\end{array}$ & $\begin{array}{l}\vec{V} \\
\left(\mathrm{~m} \mathrm{~s}^{-1}\right)\end{array}$ \\
\hline (1) Plate temperature & $329 \cdot 393$ & 0.4 & 180 & 0.2 \\
(2) Initial roughness & 368 & $0.02-0.8$ & 180 & 0.2 \\
(3) Normal load & 368 & 0.02 & $36-180$ & 0.2 \\
(4) Average speed & 368 & 0.4 & 180 & $0.1 \cdot 0.4$ \\
\hline
\end{tabular}

The following procedure was used: (1) sliding was continued until the partial formation of a transfer film on the plate; (2) curve $\mu_{x}=f(x)$ was photographed and sliding was stopped shortly afterwards; (3) the plate working surface, with the transfer film on it, was photographed; (4) profilograms of the plate surface were made in ten equidistant transverse sections on the sliding track.

I'wo correlations were investigated, according to the procedure shown schematically in Fig. 4.

(1) Local coefficient of friction $\mu_{\mathrm{x}} v$ s. average local thickness of the transfer film $\bar{g}_{\mathrm{x}}$.

(2) Local coefficient of friction $\mu_{\mathrm{x}} v \mathrm{~s}$. local area fraction of the transfer film $\delta_{\mathrm{px}}$.

In both cases the values of local coefficient of friction were read at ten positions from the curve $\mu_{\mathrm{x}}=\mathrm{f}(x)$ and related either to the corresponding local average thickness of the transfer film or the local area fraction of the film.

The local average thickness of transfer film is expressed as $\vec{g}_{\mathrm{x}}=A_{\mathrm{rx}} / W_{\mathrm{rx}}$, where $A_{\mathrm{rx}}$ is the calculated real area of the transfer film section at the position $x$, measured by means of an image processing system (IPS) and $W_{r \mathrm{x}}$ is the real length of the film section, both estimated from profilograms.

The fractional local area of transfer film $\delta_{\mathrm{px}}=A_{\mathrm{px}} / A_{\mathrm{s}}$, defined as the relative amount of area under the strip covered with a transfer film, was calculated, where $A_{\mathrm{px}}$ is the area of a film at position $x$ close to the perimeter of a wear surface of strip (also measured by IPS) and $A_{\mathrm{s}}$ is the nominal area of that surface. No correlation between the local friction coefficient and the local average thickness of a transfer film was found. It was confirmed that the thickness of the film varies for different combinations of operational variables but is approximately constant for a chosen combination, independent of the position $x$. 
a)

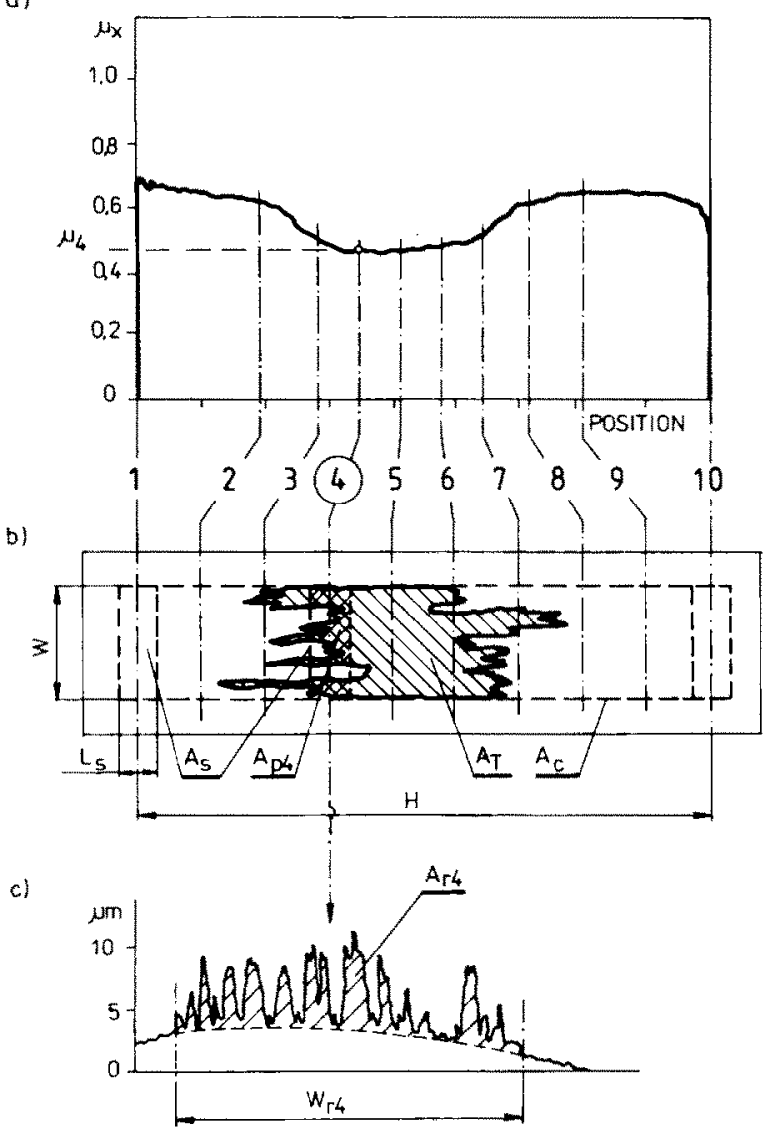

Fig. 4. Scheme of the correlation procedure for the relations (1) local friction coefficient $\mu_{\mathrm{x}}$ vs. local relative transfer film coverage $\delta_{\mathrm{px}}=A_{\mathrm{px}} / A_{\mathrm{s}}$ and (2) local friction coefficient $\mu_{\mathrm{x}}$ vs. local transfer film thickness $\overline{\mathrm{g}}_{\mathrm{x}}=A_{\mathrm{rx}} / W_{\mathrm{rx}}$ where (a) curve $\mu_{\mathrm{x}}=\mathrm{f}(x)$ is photographed from the oscilloscope, (b) the sketch represents the photograph of the transfer film on the sliding track, (c) the trace is one of the ten profilograms of the transfer film, and $A_{\mathrm{c}}$ is the total sliding area, $A_{\mathrm{T}}$ is the total transfer film area, $A_{\mathrm{s}}$ is the wear area of a strip, $A_{\mathrm{p}}$ is the local transfer film area, $A_{\mathrm{r}}$ is the local area of a transfer film transverse section and $W_{\mathrm{r}}$ is the local length of this section.

It was found, in contrast, that the local coefficient of friction could be correlated with the local area of a transfer film actually in contact with the wear surface of the strip.

Results of these analyses are presented in Fig. 5 which shows a decrease in the local friction coefficient with increase in the fractional local area of transfer film for all conditions studied. This decrease is stronger and more significant for lower values of $\delta_{\mathrm{px}}$ and seems to reach a minimum for $\delta_{\mathrm{px}}=1$ but this estimated minimum value is usually obscured by the scatter of results. It seems that this scatter may be due to action of the loose wear particles which are produced in great number during the early 

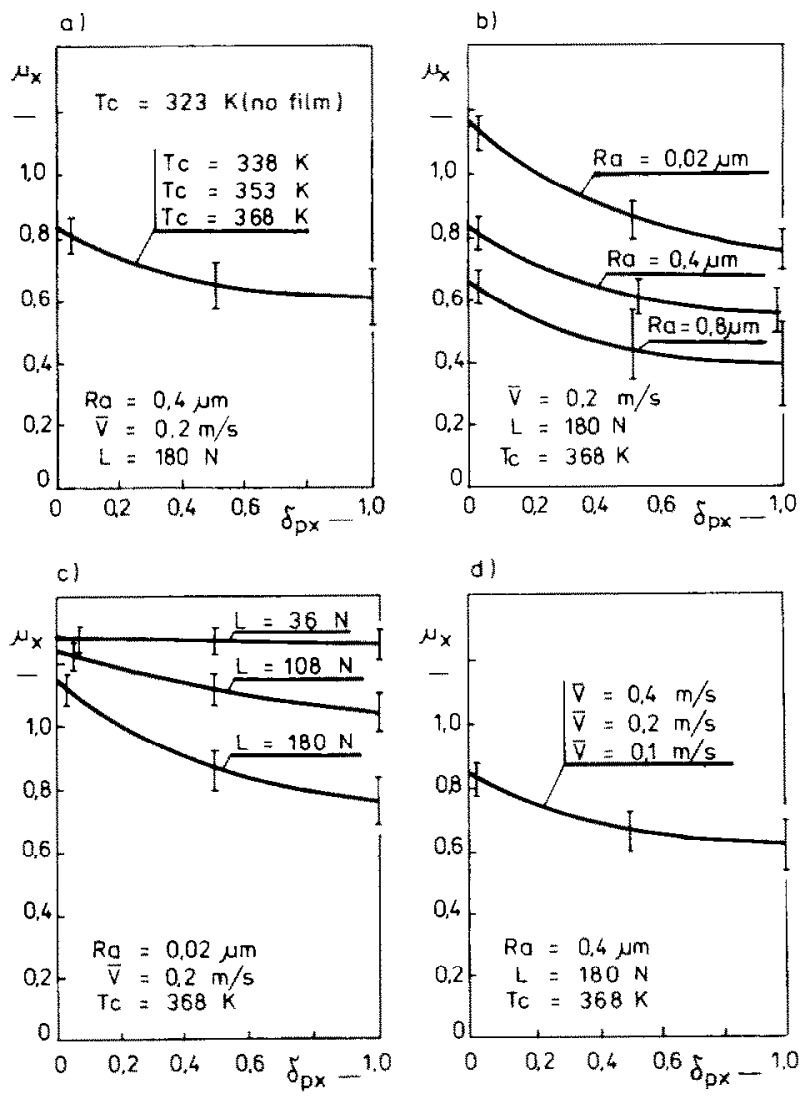

Fig. 5. The variation in the local coefficient of friction $\mu_{\mathrm{x}}$ with the dimensionless local transfer film coverage represented by the parameter $\delta_{\mathrm{px}}=A_{\mathrm{px}} / A_{\mathrm{s}}$. (a), (b), (c) and (d) illustrate the effects of plate temperature $T_{\mathrm{c}}$, counterface roughness $R_{\mathrm{a}}$, normal load $L$ and average sliding speed $\bar{V}$ respectively.

stages of sliding. Figure 5 also shows that the relation between the local friction coefficient and the local area of a transfer film is influenced significantly by the initial roughness of the counterface and by the normal load but there are no effects due to the plate temperature on sliding speed. It was observed, however, that at certain plate temperatures a transfer film did not form at all. The absence of a transfer film on the counterface for a plate temperature of $323 \mathrm{~K}\left(50^{\circ} \mathrm{C}\right)$ suggests a connection with the glass transition temperature of Nylon 6.6. In this case the sliding process did not show a variation in the local friction coefficient with the actual position of the strip, the curve $\mu_{x}=f(x)$ did not change shape with time (see Fig. 3(b)).

In the early stages of reciprocating sliding, when the transfer film begins to form, the local coefficient of friction decreases with an increase in the local area of the film. The minimum local friction coefficient is 
reached in places on the sliding track where the local area of a transfer film $\delta_{\mathrm{px}}$ is close to or equal to unity. This minimum falls with the increase in the initial roughness of the counterface and with increasing normal load. Accordingly, the mean friction coefficient should decrease with enlargement of the total transfer film area. The relation between these two quantities was investigated separately.

\subsection{Mean friction coefficient}

The variation in the mean friction coefficient was investigated as a function of the total area of transfer film, expressed non-dimensionally as $\delta_{\mathrm{T}}=A_{\mathrm{T}} / A_{\mathrm{c}}$, where $A_{\mathrm{T}}$ is the total area of film, $A_{\mathrm{c}}$ is the area of the sliding track, equal to $H\left(W+A_{s}\right)$, where $H$ is the stroke of reciprocating motion, $W$ is the width of the strip and $A_{\mathrm{s}}$ is the wear area of the strip (see Fig. 4). The relation between local coefficient of friction and the local thickness of the gradually developing transfer film was also investigated several times until steady state sliding conditions prevailed. The influence of the initial roughness of a counterface was taken into consideration and a high normal load applied to evoke a large amplitude of friction force variation. Accordingly, tests were performed for three different initial roughnesses, 0.02 , 0.1 and $0.8 \mu \mathrm{m} R_{\mathrm{a}}$, at a constant load of $180 \mathrm{~N}$, an average speed of $0.4 \mathrm{~m}$ $\mathrm{s}^{-1}$ and a plate temperature of $368 \mathrm{~K}\left(95^{\circ} \mathrm{C}\right)$. The experiment was repeated five times for each chosen roughness of the counterface. Each test started with a newly prepared sliding pair and was continued for a longer period than the preceding test. The last test was stopped only when the transfer film fully covered the sliding track and steady state conditions were reached.

The previously described correlating procedure was applied (as shown in Fig. 4) and was extended by measurements of the relative total area of transfer film $\delta_{\mathrm{T}}$ with respect to the mean coefficient of friction $\bar{\mu}$, recorded as a function of sliding time. This relation is shown in Fig. 6. As expected,

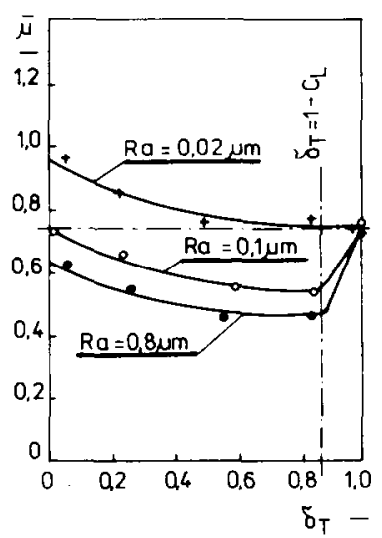

Fig. 6. The variation in the mean coefficient of friction $\bar{\mu}$ with the dimensionless area of transfer film represented by the parameter $\delta_{T}=A_{\mathrm{T}} / A_{\mathrm{c}}$ for the conditions $T_{\mathrm{c}}=368 \mathrm{~K}$, $L=180 \mathrm{~N}$ and $\bar{V}=0.4 \mathrm{~m} \mathrm{~s}^{-1}$. 
the enlargement of the transfer film produces a decrease in the mean coefficient of friction. However, only for the lowest initial roughness of a counterface, $0.02 \mu \mathrm{m} R_{\mathrm{a}}$, is this decrease monotonic and finally approaches the minimum limiting value which corresponds to the fully developed transfer film. Different relationships were observed for the initially rough counterfaces, 0.1 and $0.8 \mu \mathrm{m} R_{\mathrm{a}}$. There are two phases.

Phase 1 . Decrease in the mean friction coefficient $\bar{\mu}$ to the minimum with increase in the relative total area of transfer film $\delta_{\mathrm{T}}$, within the range $0 \leqslant \delta_{\mathrm{T}}<1-C_{\mathrm{L}}$, where $C_{\mathrm{L}}=2\left(A_{\mathrm{s}} / A_{\mathrm{c}}\right)$.

Phase 2. Increase in the mean friction coefficient $\bar{\mu}$ to the limiting value with a further increase in the transfer film area $\delta_{\mathrm{T}}$ to unity.

The final, mean coefficients of friction are approximately equal to that obtained for a smooth counterface. Two general conclusions can now be drawn.

(1) The running-in process strongly depends on the initial roughness of the counterface, resulting in the initial wear being either adhesive or abrasive. It is not clear at this stage what determines uniphase or biphase changes in the mean coefficient of friction in relation to the initial wear process.

(2) The final limiting mean coefficient of friction, corresponding to a complete coverage of the sliding track by a transfer film, seems to be independent of the initial roughness of the counterface (but dependent on the properties of the transfer film under applied load, speed and plate temperature).

\subsection{Loose wear particles}

Direct observations of the counterface during sliding and microscopical examination of the mating surfaces and the loose wear particles suggest a very important role of the latter in the running-in phase of reciprocating sliding. Various types of wear particles were found during the investigations and four basic groups, presented in Fig. 7, could be distinguished.

(1) "Needles"-- highly slender, with sharp ends (see Fig. 7(a)), but soft and viscous; could smear into a very thin transfer layer with a characteristic crescent shape. They were found only on initially smooth surfaces of the plate as a mechanical agglomeration of microscopic drops of a solidified polymer previously melted in microcontacts.

(2) "Cigars" - rather short, blunt-ended (see Fig. 7(b)); elastic rather than plastic. "Cigars" were created by rolling between the rubbing surfaces of previously irregular wear particles which were products of abrasion by the rough metallic surface on to the polymer above its glass transition temperature.

(3) "Compacts" - compact, random in shape and brittle (see Fig. $7(b))$; the result of abrasion by the rough metallic surface on to the polymer only below its glass transition temperature. They were characterized by very low adhesiveness to the metallic surface and so could not create a transfer film. 


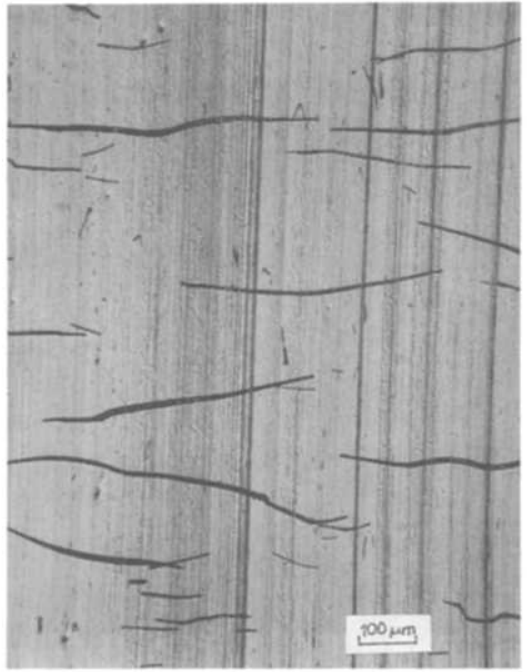

(a)

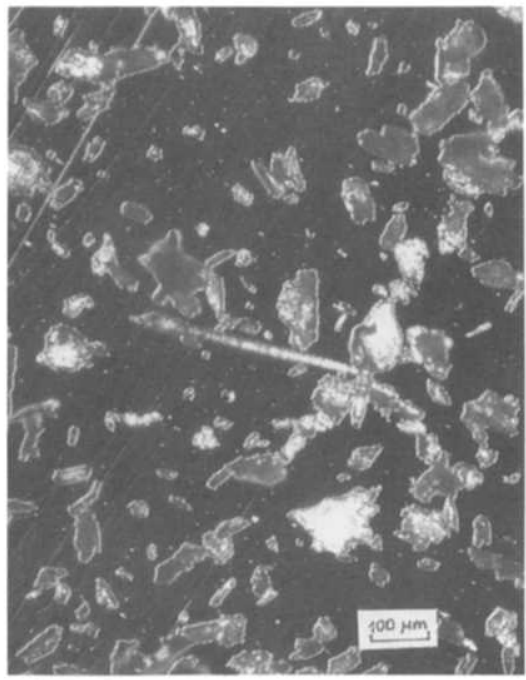

(c)

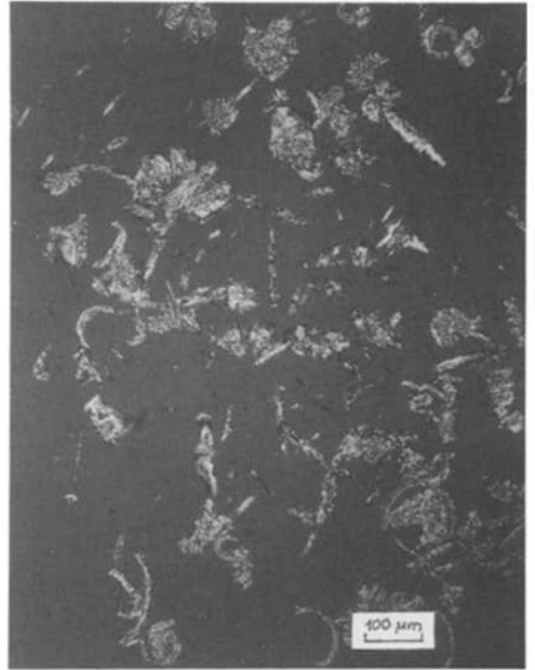

(b)

Fig. 7. Polarized micrographs of the four basic types of loose wear particles: (a) "needles"; (b) "cigars" and "compacts"; (c)"flats".

(4) "Flats" - irregular, flat and resembling floating ice (see Fig. 7(c)); usually with marks of oxidation. They only appeared when the transfer film fully covered the sliding track. These wear particles are simply the transfer film debris scraped from the ends of the sliding track. They are characteristic of the steady state phase of reciprocating sliding, independent of the initial roughness of the counterface.

The two types of roll-like wear particles, "needles" and "cigars", were mainly observed during transfer film formation in both sets of tests. When 
the films had completely formed, however, only "flat" wear debris was found, independent of the initial roughness of the counterface.

\section{Model for running-in}

It is suggested that the variation in local friction coefficient with distance, shown in Fig. 3, can be represented schematically by two types of running-in behaviour (Figs. 8(a), 9(a) and 9(b)) and a steady state regime (Figs. 8(b) and 9(c)).

\subsection{Initially adhesive wear, $0.02 \mu \mathrm{m} R_{\mathrm{a}}$}

5.1.1. Phase 1, $0 \leqslant \delta_{\mathrm{T}}<1$ (Fig. 8(a))

During the first few passes of the polymer slider over the clean smooth surface of the plate, the curve $\mu_{\mathrm{x}}=\mathrm{f}(x)$ is flat (0), but it soon changes to a concave shape with a strong local variation in the frictional force (1). It is suggested that this concavity is associated with a layer of "needles" rolling between the rubbing surfaces (1') (compare with Fig. 3(c)). The distribution of the "needles" on the sliding track is uneven. The highest concentration of the biggest needles was in the centre which corresponds to a minimum average local coefficient of friction. Conversely, there were no needles over the "turning areas" (at the ends of the stroke) where the local friction coefficient was a maximum. Only a very small number of "needles" were removed from the sliding track. The subsequent appearance of a thin transfer film in the centre of the sliding track $\left(2^{\prime}\right)$ did not result in a significant change in the average local coefficient of friction (2). Moreover, this did not suppress the local variation in the frictional force in

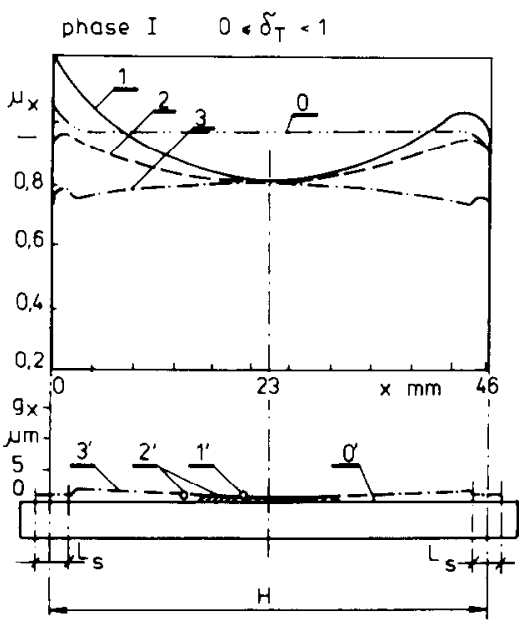

(a)

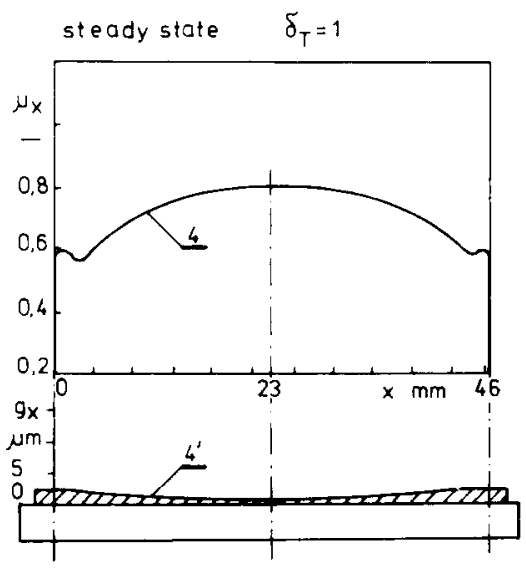

(b)

Fig. 8. The variation with stroke $H$ of the local coefficient $\mu_{\mathrm{x}}$ and transfer film thickness $g_{\mathrm{X}}$ during (a) the running-in period and (b) the steady state for initially adhesive wear. 
the centre of the sliding track. This variation seems to be due to internal flow of soft viscous "needles" in a viscous film, rather than to rolling of the "needles" above it. The areas at the ends of the stroke gradually became covered with a layer of fine "needles" which produced a gradual decrease in the local friction coefficient.

Finally, the curve $\mu_{x}=f(x)$ changed to a convex shape (3). This change corresponds to the coating of the turning areas by a transfer film $\left(3^{\prime}\right)$ and the accompanying disappearance of both "needles" and the local variation in frictional force. Running-in has now reached the steady state phase.

\subsubsection{Steady state phase, $\delta_{\mathrm{T}}=1$ (Fig. $8(b)$ )}

The curve $\mu_{\mathrm{x}}=f(x)$ is now convex (4) whereas the transfer film thickness distribution in the sliding direction $(4$ ') has a concave shape. No "ncedles" and only a very small number of floating-ice-like wear debris are being scraped from the ends of the transfer film.

\subsection{Initially abrasive wear, $0.1-0.8 \mu m R_{\mathrm{a}}$}

5.2.1. Phase 1, $0 \leqslant \delta_{\mathrm{T}}<\left(1-C_{\mathrm{L}}\right)$ (Fig. 9(a))

The curve $\mu_{x}=f(x)$, which was initially flat (0), corresponding to the evenly distributed layer of moving "cigars" rolling between the rubbing surfaces $\left(0^{\prime}\right)$, gradually changes with time into a concave shape. This concavity appears in the centre (1) and lengthens and also slightly deepens $(2,3)$ with time. This corresponds to the appearance of a transfer film in the centre of the sliding track $\left(1^{\prime}\right)$ and its further lengthening and slight thickening $\left(2^{\prime}, 3^{\prime}\right)$ (compare with Fig. 3(a)). The local friction coefficient $\mu_{\mathrm{x}}$ is lower wherever the "cigars" are rolling above the transfer film. "Cigars", which accompany the process of enlargement of the transfer film, occupy the whole sliding track and leave the sliding area in great numbers, mainly in the sliding direction.

\subsubsection{Phase 2, $\left(1-C_{\mathrm{L}}\right)<\delta_{\mathrm{T}}<1$ (Fig. 9(b))}

The curve $\mu_{\mathrm{x}}=\mathrm{f}(x)$ gradually changes from a concave to a convex shape (4) and rises (5) with time. This corresponds to coating of the turning areas by the transfer film $\left(4^{\prime}\right)$ and to a change in transfer film thickness distribution in the sliding direction to concave $\left(5^{\prime}\right)$. This process occurs with a drastic reduction in the number of "cigars" produced and eventually to their suppression. Running-in reaches the steady state phase.

\subsubsection{Steady state phase, $\delta_{\mathrm{T}}=1$ (Fig. $9(\mathrm{c})$ )}

The curve $\mu_{T}=f(x)$ is convex (6), in contrast to the concave shape of the transfer film thickness distribution in the sliding direction $\left(6^{\prime}\right)$. No "cigars" and only a very small number of "flat" wear debris are scraped from the ends of the transfer film. In spite of different running-in processes, the early phase of steady state reciprocating sliding for initially abrasive wear is similar to that for initially adhesive wear. The limiting mean friction coefficient and limiting wear rates (see Fig. 10) are approximately equal in 


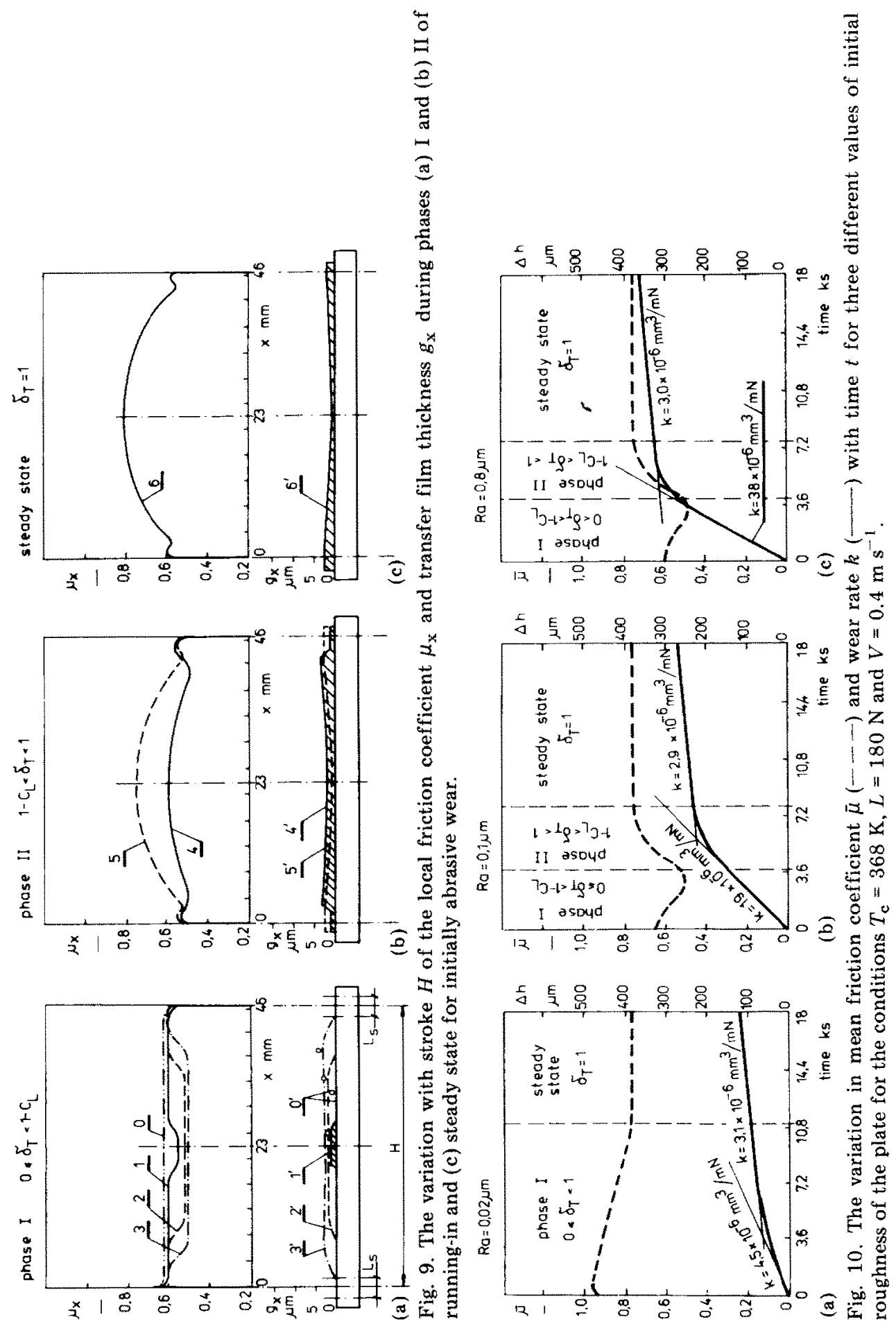


both cases. Moreover, similar correlations exist between local coefficients of friction $\mu_{\mathrm{x}}$ and average local thicknesses of a transfer film $\bar{g}_{\mathrm{x}}$. Relation of these two quantities to the magnitude of the local sliding speed $V_{\mathrm{x}}$, however, is not excluded. The controlling factor in this interaction appears to be the local temperature of the contact.

\subsection{Subprocesses in running-in}

From the observations presented above, it is postulated that four basic types of interaction occur between rubbing surfaces during running-in. Each of them possesses its own specific coefficient of friction and a specific wear rate. The latter is discussed in Section 5.5 and presented in Fig. 10. Thus the types of interaction have been called subprocesses. Their general features are as follows.

Subprocess $\mathrm{A}$ - direct contact of both rubbing surfaces without, or almost without, the influence of a third body. A single-pass condition with the specific coefficient of friction $\mu_{\mathrm{A}}$ and specific wear rate $k_{\mathrm{A}}$.

Subprocess B - existence of moveable "roll-like" wear particles between the rubbing surfaces. The specific coefficient of friction $\mu_{B}$ is lower than $\mu_{\mathrm{A}}$ and the specific wear rate $k_{\mathrm{B}}$ is lower than $k_{\mathrm{A}}$.

Subprocess $\mathrm{C}$-simultaneous presence of the moveable "roll-like" wear particles on a stationary transfer film between the rubbing surfaces. The specific coefficient of friction $\mu_{\mathrm{C}}$ is lower than $\mu_{\mathrm{B}}$ and the specific wear rate $k_{\mathrm{C}}$ is lower than $k_{\mathrm{B}}$.

Subprocess $D-$ presence of only the immoveable transfer film between the rubbing surfaces. The specific coefficient of friction $\mu_{D}$ and the specific wear rate $k_{\mathrm{D}}$ are both characteristic of the applied operational variables.

\subsection{Sequence of subprocesses}

Subprocesses occur alone or in combination on the sliding track; moreover, the position and extent of their presence change during runningin. If the specimen moves and changes position with respect to the sliding track, the transition between different subprocesses can take place two or more times in one stroke. However, the distribution of subprocesses on the counterface changes with time and it produces a specific succession of subprocesses. The initial wear process controls this sequence which is different for initially adhesive wear from that for an initially abrasive wear process.

The appearance on the counterface of the subprocesses is as follows.

For initially adhesive wear

A - subprocess exists only during the first stroke and soon disappears except in the turning areas,

B - subprocess appears after the first stroke, develops and only exists until the transfer film appears (D subprocess),

$\mathrm{B} / \mathrm{D}-$ subprocesses coexist but the increase in the $\mathrm{D}$ subprocess area decreases the B subprocess area, until,

D - subprocess is present alone. 
For initially abrasive wear

A - subprocess exists only during the first stroke and soon disappears except in the turning areas,

B - subprocess appears after the first stroke, develops and exists alone until the transfer film appears ( $\mathrm{C}$ subprocess),

B/C - subprocesses are present simultaneously but a continuous increase in area of the $C$ subprocess produces a decrease in the B subprocess area,

$(\mathrm{B} / \mathrm{C}) / \mathrm{D}$ - subprocesses exist together but gradual coating of the turning areas results in the disappearance of the couple $\mathrm{B} / \mathrm{C}$ because of suppression of the production of "cigars" until,

D - subprocess exists alone.

If the differences between the specific coefficients of friction $\mu_{A}, \mu_{B}$, $\mu_{\mathrm{C}}$ and $\mu_{\mathrm{D}}$ are sufficiently large, we can expect a distinct variation in the local coefficient of friction in relation to the specimen position. This will result in a time dependence of the shape of the curve $\mu_{x}=\mathrm{f}(x)$ and of the mean coefficient of friction. It was found that normal load and counterface roughness together control some specific coefficients of friction through the number and size of loose wear particles. At the present stage it is impossible to give a quantitative description of the variations in the mean friction coefficient with time and operating variables. However, with some degree of simplification, the mean coefficient of friction can be qualitatively expressed as a function of the relative total area of the transfer film and of the specific coefficients of friction, characteristic of the subprocesses taking part in the running-in period of reciprocating sliding. These expressions are presented in Table 2.

\subsection{Wear}

Continuous recording of the specimen height loss $\Delta h$ as a function of the sliding time $t$ during every experiment, supplements the model of running-in presented. It was found that the wear rate always decreases during running-in from a maximum, proportional to the initial roughness of the counterface, to a minimum, independent of the initial counterface roughness. The variation in wear rate, together with variations in the mean coefficient of friction with sliding time, are presented in Fig. 10 for three different initial roughnesses of the counterface.

It was confirmed that the change in wear rate is uniphase for an initially adhesive wear process, whereas it is biphase for initially abrasive wear. These phases correspond to the changes in friction. In the former, the decrease in wear rate is continuous and proportional to the increase in the total area of the transfer film. The wear rate reaches a minimum constant value when the transfer film is fully developed (see Fig. 10(a)). The change in wear rate for an initially abrasive wear process is completely different (see Figs. 10(b) and 10(c)). During phase 1, the wear rate is approximately constant, independent of the increase in the transfer film area, but proportional to the initial roughness of the counterface. In phase 2 


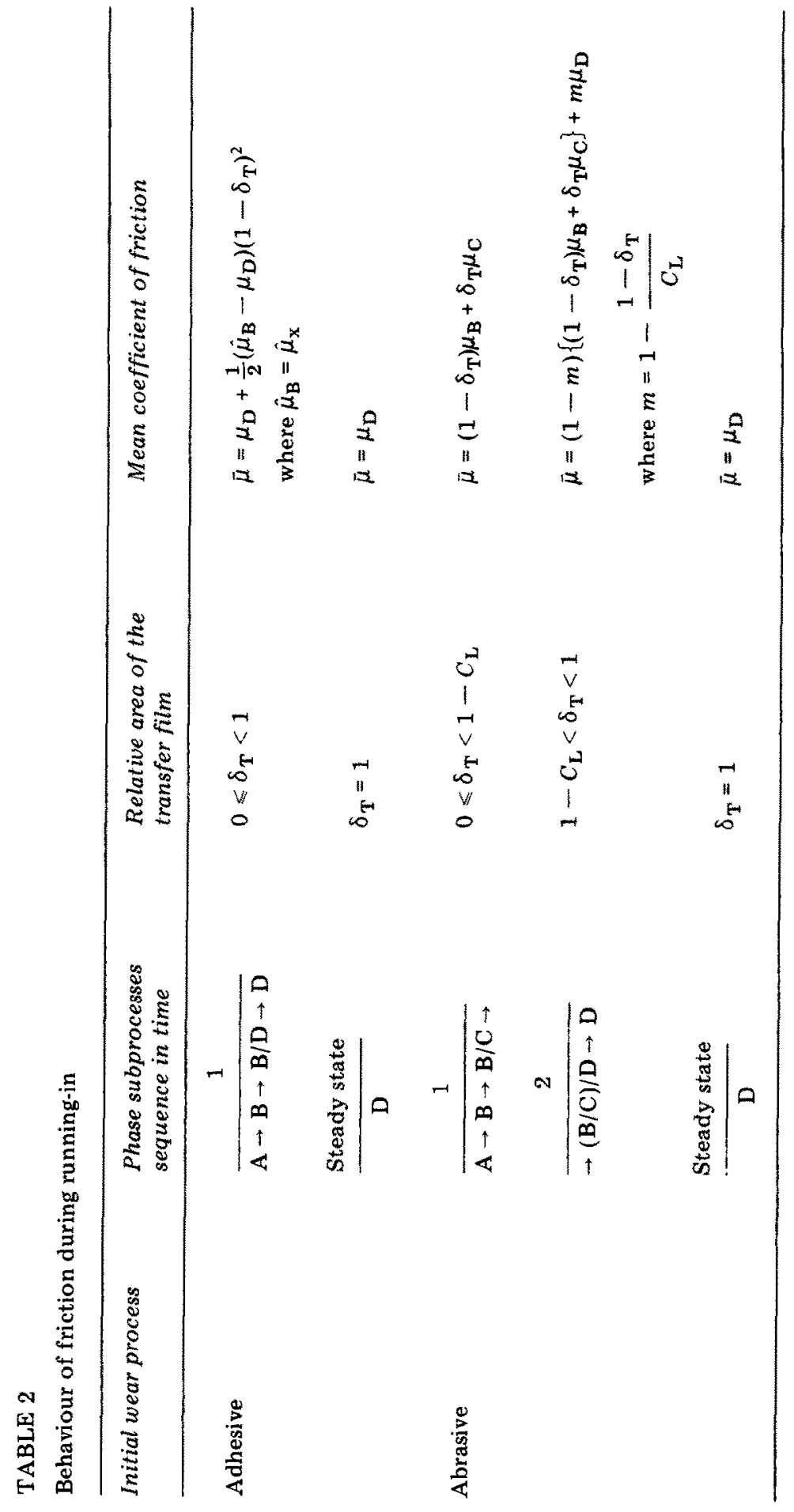


the wear rate decreases drastically with further enlargement of the transfer film over the turning areas and finally reaches a minimum constant value when the transfer film wholly covers the sliding track. The drastic decrease in wear rate in phase 2 , due to the covering of turning areas by the transfer film, implies that the main sources of wear during phase 1 are the clean turning areas whereas the rest of the sliding track, being covered by a third body separating the mating surfaces, does not contribute significantly to the total wear rate. As long as only "cigars" are rolling between the rubbing surfaces, the wear rate is constant. The appearance and further enlargement of the transfer film area produces a slight decrease in the wear rate. These observations demonstrate that each subprocess possesses its own specific wear rate. In fact the differences in the specific wear rates $k_{\mathrm{B}}, k_{\mathrm{C}}$ and $k_{\mathrm{D}}$ are small because of the similar nature of specimen wear (mainly adhesive). The deciding factors in wear are the specific wear rate $k_{\mathrm{A}}$, close to the turning areas during phase 1 , and the specific wear rate $k_{\mathrm{D}}$, for the whole sliding track in the steady state phase. The wear rate change during phase 2 is more pronounced the greater the difference between specific wear rates $k_{\mathrm{A}}$ and $k_{\mathrm{D}}$ (see Figs. $10(\mathrm{~b})$ and $10(\mathrm{c})$ ). There is also a change in wear rate for the initially adhesive wear process; however, the difference between specific wear rates, $k_{\mathrm{A}}$ and $k_{\mathrm{D}}$, is small (see Fig. $10(\mathrm{a})$ ), so the change is minor.

If the shape of a curve $\mu_{\mathrm{x}}=\mathrm{f}(x)$ is compared with the distribution of specific wear rates on the sliding track, it is apparent that, as long as the subprocess $\mathrm{A}$ is predominant in the turning areas, the maximum in friction coefficient $\hat{\mu}_{\mathrm{x}}$ occurs close to the turning points. In the steady state phase the maximum friction coefficient alters its position to the centre of the track. These observations suggest a more general rule; the maximum local wear rate occurs close to the maximum local frictional force and the reverse.

It was also found that the wear rate during running-in is greater than the transfer rate, especially when the abrasive wear process occurs initially. In the steady state phase only floating-ice-like wear debris leave the sliding track. This can be considered as a proof that the wear products of a strip are absorbed by the transfer film and are then slowly transported to the ends of the film, where, as worn-out material, they end their role in reciprocating sliding. In that case the wear rate is equal to the transfer rate.

\section{Conclusions}

(1) Running-in, with accompanying changes in friction and wear, is closely related to the build-up and spreading of a transfer film over the whole sliding track. The occurrence of roll wear particles strongly decreases the coefficient of friction.

(2) The most important factor controlling the process of running-in is the initial counterface roughness. At low normal loads the influence of the initial roughness becomes less apparent. 
(3) Four basic types of interactions between rubbing surfaces, called subprocesses, occur either alone or in combination, changing their distribution over the sliding track with time, as shown by the variation in the local coefficient of friction.

(4a) Running-in is uniphase for an initial adhesive process. The mean friction coefficient and the wear rate decrease monotonically to a minimum final value.

(4b) Running-in is biphase for an initially abrasive process.

Phase 1. The mean friction coefficient decreases while the wear rate is high and approximately constant.

Phase 2. The mean friction coefficient sharply increases to a final value while the wear rate strongly decreases to a minimum final value.

(5) Both the final mean coefficient of friction and the final wear rate are independent of the initial counterface roughness.

(6) The local coefficient of friction changes with the local subprocess while the mean coefficient of friction is related to the distribution of subprocesses over the sliding track.

(7) The maximum friction occurs in the turning areas as long as they are without a transfer film. When transfer occurs in the turning areas, the maximum shifts to the centre of the sliding track.

(8) The turning areas are found to be the main source of wear.

(9) Under steady state conditions, the correlation between the local coefficient of friction, the local thickness of the transfer film and the local magnitude of the sliding speed seems to be good. It is probable that the local temperature in the contact zone is the controlling factor in the interaction process.

\section{Acknowledgment}

One of the authors (Z.Z.) wishes to acknowledge the hospitality of the Tribology Group of the Mechanical Engineering Department of the University of Twente and the award of a research fellowship which enabled this work to be carried out.

\section{References}

1 V. K. Jain and S. Bahadur, Material transfer in polymer-polymer sliding, Wear, 46 (1978) 177 - 201.

2 S. K. Rhee and K. C. Ludema, Mechanisms of formation of polymeric transfer films, Wear, 46 (1978) $231-240$.

3 V. K. Jain and S. Bahadur, Surface topography changes in polymer-metal sliding, J. Lubr. Technol., 102 (1980) $520-525$.

4 J. R. Atkinson, K. J. Brown and D. Dowson, The wear of ultrahigh molecular weight polyethylene, Part I, J. Lubr. Technol, 100 (1978) $208-218$. 
$5 \mathrm{~J}$. K. Lancaster, Geometrical effects on the wear of polymers and carbons, J. Lubr. Technol., (April 1975) $187-194$.

$6 \mathrm{~K}$. Tanaka, Transfer of semicrystalline polymers sliding against a smooth steel surface, Wear, 75 (1982) 183 - 199.

7 K. J. Brown, J. R. Atkinson and D. Dowson, The wear of ultrahigh molecular weight polyethylene, part II, J. Lubr. Technol, 104 (1982) $17-22$.

8 R. L. Fusaro, Friction, wear, transfer, and wear surface morphology of UHMWPE, ASLE Trans. 28.1 (1985) 1 - 10.

9 J. K. Lancaster, D. Play, H, Godet, A. P. Verall and R. Waghorne, Third body formation and the wear of PTFE fibre based dry bearings, J. Lubr. Technol, 102 (1980) $236-246$.

10 M. K. Kar and S. Bahadur, Micromechanism of wear at polymer-metal sliding interface, Wear, 46 (1978) $189-202$.

$11 \mathrm{P}$. H. Vroegop and R. Bosma, Subsurface melting of Nylon by friction-induced vibrations, Wear, $104(1985) 31-47$.

\section{Appendix A}

\section{A.1. Evaluation of the mean frictional force in a reciprocating tribometer}

In a reciprocating machine, operating at constant frequency, the relative movement of the sliding bodies can be described as a periodic function and may be written as a Fourier series. The frictional force changes sign with the direction of sliding and can, in general, be approximated by the sum of a periodic function $F$ and a random disturbance $R$. Both functions have expectancy zero.

The sliding speed $V$ as a function of time can be written as

$$
V(t)=\sum_{n=1}^{\infty} n a_{n} \omega \cos \left(n \omega t+\gamma_{n}\right)
$$

where $a_{n}$ represents the Fourier coefficients and $\omega$ is the angular frequency of the reciprocating motion. The frictional force $F$ can be written as

$F(t)=\sum_{m=1}^{\infty} F_{m} \cos \left(m \omega t+\gamma_{m}\right)+\mathrm{R}$

The sliding speed and frictional force should be in phase since $F(t)$ changes sign with speed, i.e. at the extremes of the displacement.

Important aspects of the friction signal are the extreme value during one cycle and the frictional work $W$ done per cycle. The latter follows from integration of the frictional force with respect to the displacement over one cycle, i.e.

$$
W=\int_{-\pi}^{\pi} F V \mathrm{~d}(\omega t)
$$

Substitution of eqns. (A1) and (A2) into eqn. (A3) and evaluation of the integral using Fourier theory leads to the result 
$W=\pi \sum_{n=1}^{\infty}\left(n^{2} a_{n} F_{n}\right)$

In the $\mathrm{S}$ tribometer the motion is sinusoidal, i.e. $a_{n}=0$ for $n \neq 1$, so the frictional work follows from

$W=\pi a F_{1}$

where $a=a_{1}$ is the amplitude of the displacement and $F_{1}$ is the first Fourier coefficient of the friction signal.

The mean coefficient of friction applied in this paper is defined by the frictional work done, divided by the (constant) normal force and by the length $4 a$ of one cycle. Equation (A5) shows that this value is proportional to the first Fourier coefficient of the friction signal. It can be measured using a frequency analyser or using simply an adjustable bandpass filter.

Calibration is performed by feeding a square wave to the measurement circuits for both the maximum and the mean coefficient of friction and by adjusting the amplification for the mean value until it equals the maximum value.

It should be noted that this mean coefficient of friction is not affected by the random part $R$, as would have been the case when using the r.m.s. value of the friction signal. Averaging is performed with respect to the sliding length, not to the time of sliding. 